\title{
Pasternak Model Formulation of Elastic Displacements in the Case of a Rigid Circular Foundation
}

\author{
Hideaki Tanahashi
}

Director, TASS Design Institute, Researcher, Disaster Prevention Research Institute, Kyoto University, Japan

\begin{abstract}
The classical Winkler model has often been used in many engineering fields. However, it has some shortcomings, because the adjacent displacements are discontinuous. In this paper, the Pasternak model — an improved continuum model - is applied to the surface displacement distribution analysis of an elastic layer with a finite depth in an axisymmetric condition. The parameters of the Pasternak model are determined by comparing its surface displacement distributions with those of a finite element analysis. On the basis of the results, the author proposes the non-dimensional characteristic values for the Pasternak model with a simple closed-form formulation of surface displacement distributions in the case of a rigid circular foundation on an elastic layer with a finite depth. The author then discusses the accuracy of the approximate formulation and the limits of its applicability.
\end{abstract}

Keywords: Pasternak model; Winkler model; elasticity; axisymmetric condition; coefficient of subgrade reaction

\section{Introduction}

The Winkler model (Winkler, 1867) has been used effectively in geotechnical analyses of a beam on an elastic soil layer and pile behaviors resulting from horizontal loads, etc. The model originated from "Winkler's hypothesis," which states that the deflection at any point on the surface of an elastic continuum is proportional only to the load being applied to the surface and is independent of the load applied to any other points on the surface (Winkler, 1867). This hypothesis leads to a mechanical model of a continuum that is assumed to consist of mutually independent vertical linear springs. However, it has some shortcomings due to the discontinuity of the adjacent displacements.

In order to overcome such shortcomings, a number of researchers have proposed improved or refined models (Filonenko-Borodich, 1940; Hetényi, 1946; Pasternak, 1954; Reissner, 1958; Vlasov et al., 1960; Kerr, 1964; and Loof, 1965). These are often called two-parameter models because, in addition to the first parameter, the coefficient of subgrade reaction, they have a second parameter that shows the continuity of adjacent displacements.

Pasternak (1954) introduced shear interaction between adjacent Winkler's spring elements. Kerr (1964) regarded Pasternak's mechanical model as a

\footnotetext{
*Contact Author: Hideaki Tanahashi, Suzuki Laboratory, Disaster Prevention Research Institute, Kyoto University, Gokasho, Uji, 611-0011, Japan

Tel: +81-774-38-4050 Fax: +81-774-38-4055

e-mail: tana@zeisei.dpri.kyoto-u.ac.jp

(Received October 6, 2006 ; accepted February 16, 2007)
}

shear layer on the Winkler model and used the term "Pasternak model" to refer to a generalized foundation model. The Pasternak model (hereinafter abbreviated as PM) is used here in the same manner.

Since two-parameter models naturally require both parameters for practical applications, methods for determining the parameters have been discussed for the past several decades. Pasternak himself suggested using plate loading tests for two parameter evaluations, but he didn't give the parameters actual values. Vlasov et al., (1960) made certain assumptions in order to formulate two parameters for the Vlasov model. This was a significant contribution to determining the parameters of the PM. Furthermore, Jones et al., (1977) established a rigorous theoretical base for the shape function of the vertical displacement profile suggested by Vlasov et al., (1960).

Vallabhan et al., (1988) modified the Vlasov model and showed solutions for an elastic beam on an elastic layer. Using an iterative procedure, they also accurately solved the equations for two parameters established by Jones et al., (1977). In their solutions, however, the displacements tend to be smaller than those of a continuum. In the author's view, these tendencies toward underestimation come mainly from Vlasov's assumptions, even if the solutions are reasonably accurate mathematically. These tendencies in the approximate solutions may be unavoidable to some extent. However, their accuracy has yet to be clarified.

Although the finite element method (FEM) is very useful for numerical analyses in a continuum, it can never provide closed-form solutions. Therefore, in previous studies for practical applications, the author selected the PM for closed-form solutions of surface 
displacements on an elastic layer and proposed parameters for actual calculations (Tanahashi, 2000a, $2000 \mathrm{~b}$ ). The parameters of the PM are reduced to a non-dimensional characteristic value $\gamma H$ of the system. These recommended values for the constants were derived by comparing the surface displacement distributions of the PM with those of the FEM. Cases investigated to date have been limited to those without a foundation on the elastic layer (Tanahashi, 2000a: two-dimensional plane strain condition; Tanahashi, 2000b: axisymmetric condition) and with uniform flexible vertical loads distributed directly on the surface.

An extension of the author's previous investigations, this paper proposes surface displacements and the non-dimensional characteristic values $\gamma H$ in the case of a rigid circular foundation on an elastic layer with a finite depth. This paper also discusses the accuracy of the approximate formulation and the limits of its applicability. Such a formulation is very useful and convenient for practical applications such as preliminary analyses or order checking of surface displacement distributions in the case of rigid foundations on elastic continua.

\section{Governing Equation of the PM}

Let us consider an elastic layer that is isotropic, homogeneous, and has a depth $H$, Young's modulus $E$, and Poisson's ratio $v$ on a rough, rigid base (Fig.1.). A rigid circular foundation with radius $R$ lies on the surface.

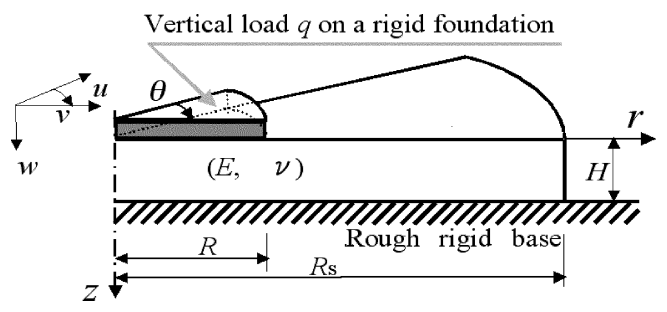

Fig.1. Analytical Model

The governing equation for surface displacements with no foundation in an axisymmetric condition has already been obtained based on the variational principles (Tanahashi, 2000b). However, the displacements are constrained by the rigid foundation in the present case. Therefore, a governing equation can be established using the Lagrange multiplier method (Washizu, 1975). Since the displacement beneath the rigid foundation is constant and assumed to be $C$, the total potential energy $\Pi$ of the system can be introduced using a Lagrange multiplier $\lambda$ as follows.

$\Pi=\int_{0}^{\infty} \int_{0}^{2 \pi} \int_{0}^{H} \frac{1}{2}\left(\sigma_{r} \varepsilon_{r}+\sigma_{\theta} \varepsilon_{\theta}+\sigma_{z} \varepsilon_{z}+\tau_{r z} \gamma_{z z}\right) d z d \theta r d r$

$-\int_{0}^{R} \int_{0}^{2 \pi} q w(r, \theta, 0) d \theta r d r+\int_{0}^{R} \int_{0}^{2 \pi} \lambda[w(r, \theta, 0)-C] d \theta r d r$

where $\sigma_{r}, \sigma_{\theta}, \sigma_{z}, \tau_{r z}, \varepsilon_{r}, \varepsilon_{\theta}, \varepsilon_{z}, \gamma_{r z}$ are the stresses and corresponding strains of a small element of the continuum, and $u, v, w$ are the radial, peripheral, and vertical displacements, respectively, of a point $(r, \theta, z)$ in the cylindrical coordinate.

The constitutive equations in an axisymmetric condition are as follows.

$\left\{\begin{array}{l}\sigma_{r} \\ \sigma_{\theta} \\ \sigma_{z} \\ \tau_{r z}\end{array}\right\}=\frac{(1-v) E}{(1+v)(1-2 v)}\left(\begin{array}{cccc}1 & \frac{v}{1-v} & \frac{v}{1-v} & 0 \\ \frac{v}{1-v} & 1 & \frac{v}{1-v} & 0 \\ \frac{v}{1-v} & \frac{v}{1-v} & 1 & 0 \\ 0 & 0 & 0 & \frac{1-2 v}{2(1-v)}\end{array}\right)\left\{\begin{array}{c}\varepsilon_{r} \\ \varepsilon_{\theta} \\ \varepsilon_{z} \\ \gamma_{r z}\end{array}\right\}$

where the peripheral displacement $v=0$ in an axisymmetric condition, and the following relations are described.

$\varepsilon_{r}=\frac{\partial u}{\partial r}, \varepsilon_{\theta}=\frac{u}{r}, \varepsilon_{z}=\frac{\partial w}{\partial z}, \gamma_{r z}=\frac{\partial u}{\partial z}+\frac{\partial w}{\partial r}$

For an approximate analysis, the following assumptions proposed by Vlasov are made. (Vlasov et al., 1960)

1) The radial displacement $u$ is assumed to be zero everywhere in the continuum, because the radial displacements are negligible in comparison with the vertical displacements under vertical load conditions.

2) The vertical displacement $w$ is expressed using a vertical surface displacement $W(r)$ and a shape function $\phi(z)$ as

$$
w(r, 0, z)=W(r) \phi(z)
$$

where $\phi(0)=1, \phi(H)=0, w(r, 0,0)=W(r)$.

Inserting Eqs. (2) and (4) into Eq. (1) yields Eq. (5).

$$
\begin{aligned}
\Pi & =\int_{0}^{\infty} \int_{0}^{2 \pi} \int_{0}^{H}\left[\frac{(1-v) E}{2(1+v)(1-2 v)}\left(\frac{d \phi}{d z}\right)^{2} W^{2}+\frac{E}{4(1+v)} \phi^{2}\left(\frac{d W}{d r}\right)^{2}\right] d z d \theta r d r \\
& -\int_{0}^{R} \int_{0}^{2 \pi} q W d \theta r d r+\int_{0}^{R} \int_{0}^{2 \pi} \lambda(W-C) d \theta r d r
\end{aligned}
$$

Here, the following governing equation is obtained by minimizing the function $\Pi$ concerning $W$ and $\lambda$ on the basis of the variational principles

$$
\begin{array}{ll}
k W_{1}-G\left(\frac{d^{2} W_{1}}{d r^{2}}+\frac{1}{r} \frac{d W_{1}}{d r}\right)=q-\lambda & : 0 \leq r \leq R \\
W_{1}-C=0 & \\
k W_{2}-G\left(\frac{d^{2} W_{2}}{d r^{2}}+\frac{1}{r} \frac{d W_{2}}{d r}\right)=0 & : r>R
\end{array}
$$

where

$$
\begin{aligned}
\gamma & =\sqrt{\frac{k}{G}} \\
k & =\frac{(1-v) E}{(1+v)(1-2 v) H} \int_{0}^{H}\left(\frac{d \phi}{d z}\right)^{2} d z \\
G & =\frac{E}{2(1+v)} \int_{0}^{H} \phi^{2} d z
\end{aligned}
$$


$\gamma:$ characteristic value of the system

$k$ : coefficient of subgrade reaction

$G$ : shear stiffness of the continuum in the vertical direction, which differs from the shear modulus of the continuum. When this is zero, $\gamma$ becomes infinity and the model corresponds to the Winkler model.

Eq. (7) shows the theoretical bases of the two parameters in an elastic layer with a finite depth.

\section{Parameters and Shape Functions of the PM}

Vlasov et al., (1960) assumed the shape function $\phi$ in a thin layer to be as follows.

$$
\phi(z)=1-z / H
$$

They assumed it in a deep layer to be

$$
\phi(z)=\frac{\sinh \eta(1-z / H)}{\sinh \eta}
$$

where $\eta$ is a parameter that determines the decrease in the vertical displacements. However, Vlasov et al., (1960) did not rigorously clarify the theoretical bases of these shape functions.

Herein, Eq. (9) generally includes Eq. (8), because when $\eta=0$, Eq. (9) coincides with Eq. (8).

When vertical loads are distributed across a surface, i.e., $R=\infty$, and the strains are constant throughout the layer, then Eq. (7) yields Eq. (10)

$$
\begin{aligned}
k_{0} & =\frac{(1-v) E}{(1+v)(1-2 v) H} \\
G_{0} & =\frac{E H}{6(1+v)}
\end{aligned}
$$

where

$k_{0}$ : fundamental coefficient of subgrade reaction

$G_{0}$ : fundamental shear stiffness

In general cases, using Eq. (10), Eq. (11) is obtained as follows.

$$
\begin{aligned}
& k=\frac{(1-v) E}{(1+v)(1-2 v) H} \eta \frac{\sinh \eta \cosh \eta+\eta}{2 \sinh ^{2} \eta}=k_{0} \varphi_{k} \\
& G=\frac{E H}{2(1+v)} \frac{1}{\eta} \frac{\sinh \eta \cosh \eta-\eta}{2 \sinh ^{2} \eta}=3 G_{0} \varphi_{G}
\end{aligned}
$$

where

$$
\begin{aligned}
\varphi_{k} & =\eta \frac{\sinh \eta \cosh \eta+\eta}{2 \sinh ^{2} \eta} \\
\varphi_{G} & =\frac{1}{\eta} \frac{\sinh \eta \cosh \eta-\eta}{2 \sinh ^{2} \eta}
\end{aligned}
$$

Accordingly

$$
\gamma=\sqrt{\frac{k}{G}}=\sqrt{\frac{k_{0} \varphi_{k}}{3 G_{0} \varphi_{G}}}=\sqrt{\frac{2(1-v)}{(1-2 v) H^{2}} \frac{\varphi_{k}}{\varphi_{G}}}=\sqrt{\frac{2(1-v)}{(1-2 v)}} \frac{\Phi}{H}
$$

where

$$
\Phi=\sqrt{\frac{\varphi_{k}}{\varphi_{G}}}=\eta \sqrt{\frac{\sinh \eta \cosh \eta+\eta}{\sinh \eta \cosh \eta-\eta}}
$$

It is emphasized that $\gamma H$ depends upon Poisson's ratios and the function $\Phi$ and is a constant value (3.24 in the case of $v=0.3$ and $\eta \rightarrow 0$ ).

Jones et al., (1977) derived the differential equation, Eq. (15), of $\phi$ by minimizing the total potential energy $\Pi$ of Eq. (5) concerning $\phi$.

$$
-m \frac{d^{2} \phi}{d z^{2}}+n \phi=0
$$

where

$$
\left.\begin{array}{l}
m=\frac{(1-v) E}{(1+v)(1-2 v)} \int_{0}^{\infty} W^{2} r d r \\
n=\frac{E}{2(1+v)} \int_{0}^{\infty}\left(\frac{d W}{d r}\right)^{2} r d r
\end{array}\right\}
$$

By introducing parameter $\eta$ as

$\frac{n}{m}=\left(\frac{\eta}{H}\right)^{2}$

Eq. (15) yields Eq. (18) as

$$
\frac{d^{2} \phi}{d z^{2}}-\left(\frac{\eta}{H}\right)^{2} \phi=0
$$

Eq. (16) means that $m$ and $n$ depend on the surface displacement distribution $W$.

Jones et al., (1977) solved Eq. (18) with the boundary conditions $\phi(0)=1$ and $\phi(H)=0$, then showed that the solution is exactly the same as the shape function in Eq. (9). Thus, they established the theoretical bases of the shape function suggested by Vlasov et al., (1960).

\section{The PM Solution}

$W_{2}$ becomes zero when $r$ increases toward infinity and the compatibility conditions of $W_{2}$ are as follows.

$$
\left.\begin{array}{l}
W_{2}(R)=C \\
W_{2}(\infty)=0
\end{array}\right\}
$$

Also, the equilibrium between the vertical loads and the reactions of the base is as follows.

$$
\pi R^{2} q=\int_{0}^{\infty} \int_{0}^{2 \pi} k W d \theta r d r
$$

Using Eqs. (19) and (20), the following surface displacements are obtained

$$
\begin{array}{ll}
0 \leq r \leq R: & W_{1}=S \frac{K_{0}(\gamma R)}{K_{2}(\gamma R)} \\
r>R: & W_{2}=S \frac{K_{0}(\gamma r)}{K_{2}(\gamma R)}
\end{array}
$$

where $K_{0}$ and $K_{2}$ are zero-order and second-order modified Bessel functions of the second kind, respectively. $S$ is expressed with $S_{0}$, which denotes a fundamental vertical displacement.

$$
S=\frac{q}{k}=\frac{q}{k_{0} \varphi_{k}}=\frac{(1+v)(1-2 v) q H}{(1-v) E \varphi_{k}}=\frac{S_{0}}{\varphi_{k}}
$$

$S_{0}$ is the same as the displacement in the case of $R \rightarrow$ $\infty$ and is of the following form. 
$S_{0}=\frac{q}{k_{0}}=\frac{(1+v)(1-2 v) q H}{(1-v) E}$

The unknown $\lambda$ of the Lagrange multiplier is as follows.

$$
\lambda=\left[1-\frac{K_{0}(\gamma R)}{K_{2}(\gamma R)}\right] q
$$

This refers to a reaction underneath the rigid foundation, which does not appear in the base. It also corresponds to the concentrated edge reaction in the case of a rigid plate on an elastic half space.

The solution Eq. (21) was obtained first by Pasternak (1954), then by Vlasov et al., (1960), using a slightly different mathematical expression.

\section{Determination of Parameters}

To determine the parameters in Eq. (7), the shape function $\phi$ must be determined. In general, however, the distribution of vertical displacements cannot be expressed with only one shape function because the distribution depends on the load condition and the position of the layer, etc.

Therefore, the problem is how to determine $\phi$ or $\eta$. Eq. (16) needs a surface displacement distribution. Thus, Vallabhan et al., (1988) proposed an iterative method to solve Eqs. (6) and (15) simultaneously.

Here, an iterative method based on that of Vallabhan et al., is applied to obtain the parameter $\gamma$ as follows. In the case of some values of $R / H$, if the first value $\gamma_{1}$ $=0.5$, for example, then the distribution $W_{1}$ of surface displacements is calculated from Eq. (21). $\eta_{1}$ is obtained from Eq. (17) through the integration of Eq. (16). $\gamma_{2}$ is then obtained from Eqs. (13) and (14). This
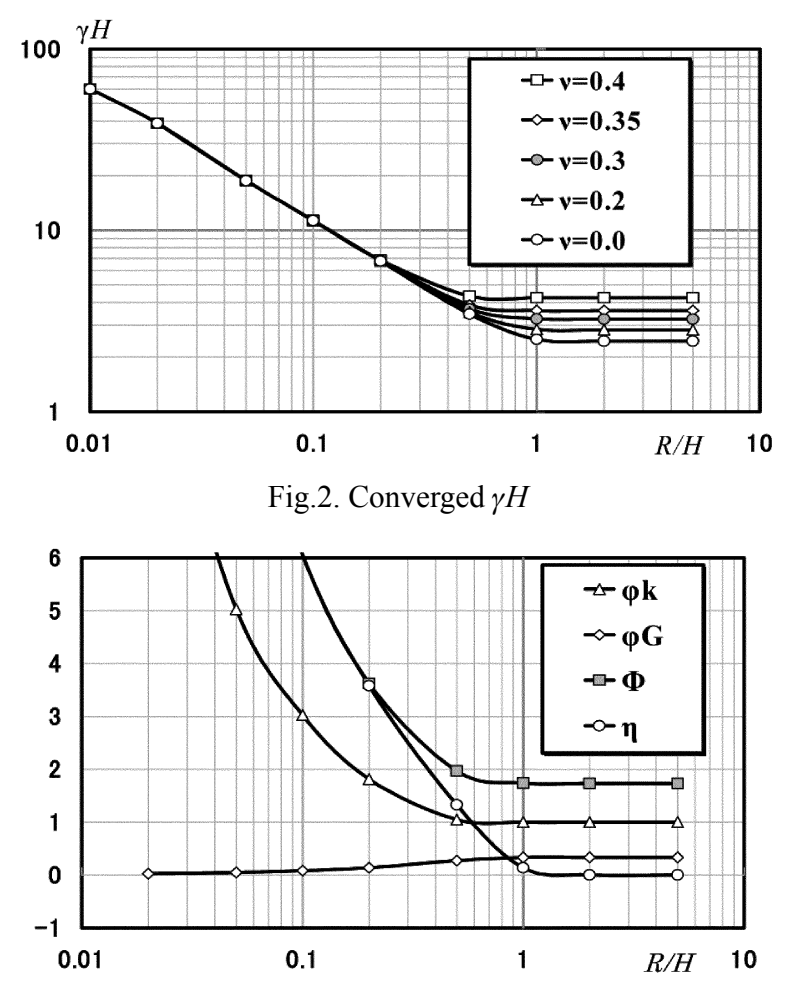

Fig.3. Converged Parameters $(v=0.3)$ procedure is continued until $\left|\gamma_{n+1}-\gamma_{n}\right|$ is minimized. Thus the final $\gamma$ is determined. Note that this procedure does not depend on the unknown displacement $S$.

$\gamma$ is obtained by this procedure in combinations of $R / H$ and $v$. Non-dimensional characteristic values $\gamma H$ are presented in Fig.2. Fig.3. shows an example of the distributions of parameters $\varphi_{k}, \varphi_{G}, \Phi$, and $\eta$ in the case of $v=0.3$.

In all cases, the minimized $\left|\gamma_{\mathrm{n}+1}-\gamma_{\mathrm{n}}\right|$ were less than $10^{-6}$. Figs.2. and 3. show that $\gamma H$ becomes constant when $R / H$ increases. Thus, $\gamma H$ is supposed to be constant even if $R / H$ becomes larger than 5 . On the other hand, if $R / H$ becomes smaller than $0.1, \gamma H$ increases greatly and becomes the same value in every $v$.

In the sense that the total potential energy $\Pi$ is minimized, the displacement solutions obtained through this procedure are the best approximations that can be derived from the assumption expressed in Eq. (4). However, such solutions for an elastic layer are not necessarily better than FEM solutions, as shown in the next chapter. In any case, it is interesting to note that the values of $\gamma H$ are distributed in a relatively narrow range - between 4 and 6 - if $R / H$ is larger than 0.2 .

\section{Comparison with FEM Solutions}

The PM displacements expressed in Eq. (21) with $\gamma$ obtained by the iterative method are compared with those obtained by FEM for uniformly distributed vertical loads on a circular rigid foundation. FEM is, of course, an approximate numerical analysis, the accuracy of which depends on the mesh geometry and boundary conditions, etc. Therefore, it was necessary to first confirm that the FEM solutions were relatively and reasonably accurate. This was done as follows.

The analyzed object was a foundation with a radius $R$ on an elastic layer having a depth of $10 \mathrm{~m}$, with Young's modulus $E=1 \mathrm{MPa}$ and Poisson's ratio $v$. A uniformly distributed vertical load $q=100 \mathrm{kPa}$ was applied to the foundation. A total of 48 cases were calculated using various combinations of radii $(R=0.2$, $0.5,1,2,5,10,20,50 \mathrm{~m})$ and Poisson's ratios $(v=0,0.1$, $0.2,0.3,0.35,0.4)$.

Axisymmetric analyses of FEM have the following boundary conditions. 1) The horizontal boundary interface between the layer and the rigid base is fixed. 2) The radii $R s=50 \mathrm{~m}$ and $100 \mathrm{~m}$ of the layer are taken into account for $R \leqq 20 \mathrm{~m}$ and $50 \mathrm{~m}$, respectively, in FEM analyses in which the boundary conditions have little influence. In such cases, the vertical peripheral boundary at $r=R s$ is fixed only in the horizontal direction. Rectangular-type elements are used. The element meshes are created so as to be finer in the areas of higher stress concentration. These meshes have been confirmed to be fine enough to ensure accuracy within a $1 \%$ margin of error in the preliminary analysis. A sample of the mesh geometry used for this analysis is shown in Fig.4. 
The surface displacement curves for the FEM and the PM with $\gamma H$ obtained by the iterative method are shown in Figs.5., 6., and 7. These figures show that the PM has smaller displacements and smaller slopes of

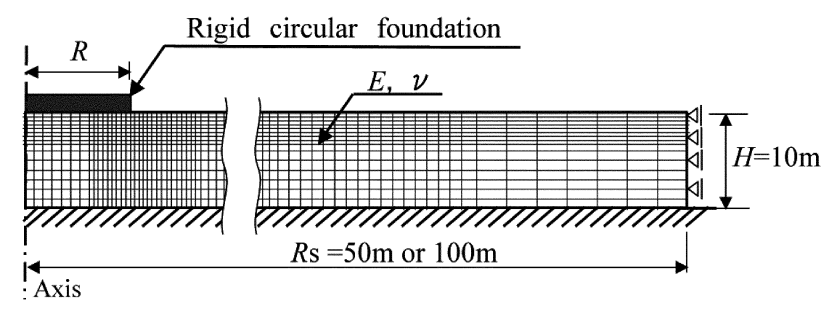

Fig.4. Sample of Mesh Geometry for FEM

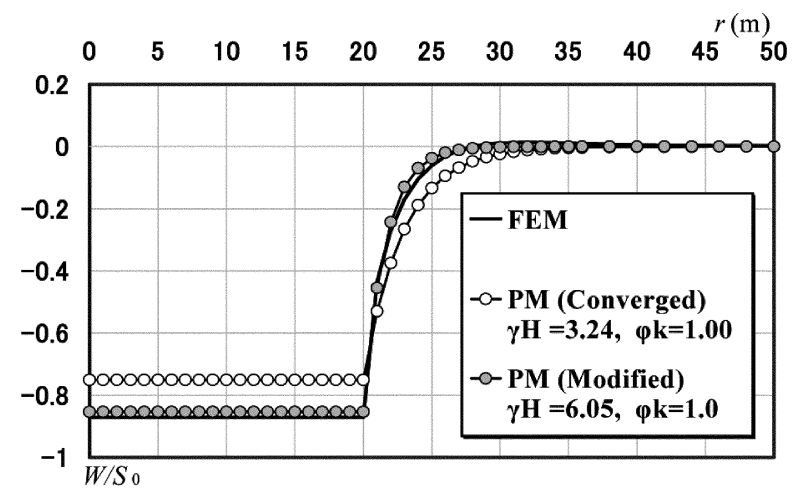

Fig.5. Comparison of PM with FEM $(R=20 \mathrm{~m}, R / H=2, v=0.3)$

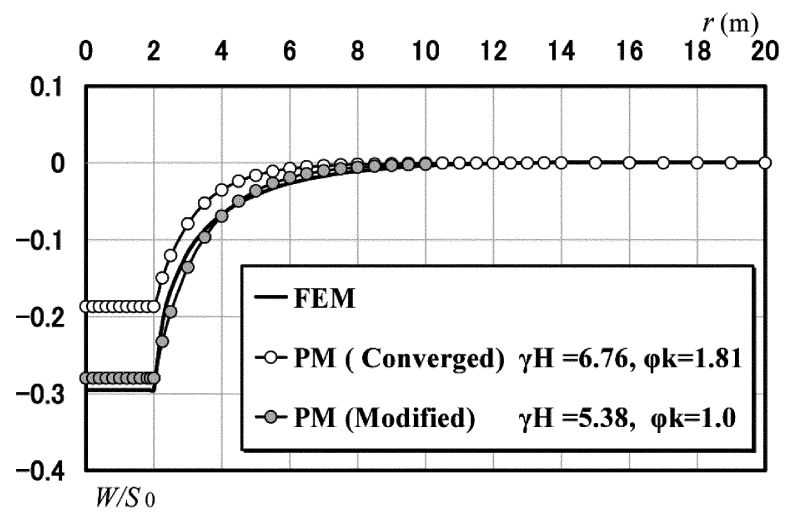

Fig.6. Comparison of PM with FEM $(R=2 \mathrm{~m}, R / H=0.2, v=0.3)$

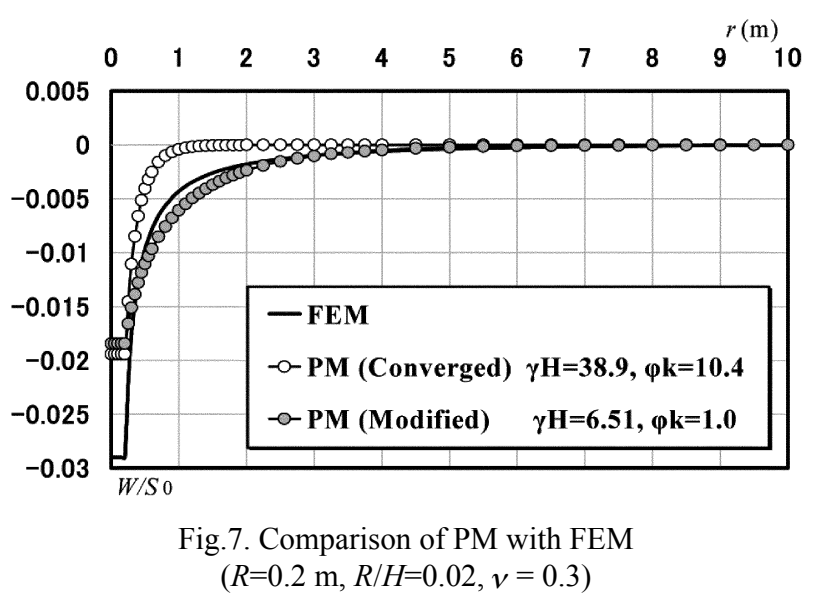

surface displacements than the FEM. These tendencies were pointed out earlier by Vallabhan et al., (1988, 1991). Although the errors due to Vlasov's assumptions are unavoidable to some extent, they are large enough that accuracy must be improved and the parameters modified within reason.

\section{Achieving a Closer Approximation}

The solutions obtained from the PM can be improved in various ways, such as by changing some of the parameters $\varphi_{k}, \varphi_{G}, \Phi, \eta$ or $\gamma$. Introducing a new parameter or creating a new mechanical model is another possible option. For convenient and practical applications, however, any modification should be within the two-parameter model. In this study, one simple modification, $\varphi_{k}=1$ is fixed. This is reasonable because the coefficient of the subgrade reaction is taken as $k_{0}$ in Eq. (10), which is the same as that of the Winkler model. $\gamma$ is then modified to minimize the weighted least-squares sum $\Delta E$ of the displacement errors as follows.

$$
\Delta E=\sum_{i=0}^{n}\left(W_{P i}-W_{F i}\right)^{2} r_{i}\left(r_{i+1}-r_{i}\right)
$$

where

$$
\begin{aligned}
& W_{P i}: \text { surface displacement of PM at } r=r_{i} \\
& W_{F i}: \text { surface displacement of FEM at } r=r_{i}
\end{aligned}
$$

The modified displacements of the PM are shown along with the modified values of $\gamma H$ in Figs.5., 6., and 7. In the cases of $R / H=2$ and 0.2 , displacement curves are greatly improved. In the case of $R / H=0.02$, many improvements were attempted, but considerable errors remained in the end.

If Poisson's ratio increases, then heaving surface displacements increase in the peripheral area outside the foundation and the PM generates more errors. These consequences occur because the modified Bessel functions $K_{0}, K_{2}$ cannot accept negative values. Also, as $R / H$ decreases to less than 0.1 , the sizes of errors increase greatly.

Non-dimensional characteristic values $\gamma H$ were calculated in 48 cases. The results are shown in Table 1. and Fig.8. Table 1. shows two types of $\gamma H$. The upper lines show the results obtained by the above-mentioned weighted least-squares sum method, while the lower lines show those obtained by fitting the foundation displacements to those of the FEM solutions. The values in the upper lines are useful for considering displacement curves, e.g., slopes or differential settlements of ground surfaces. Those in the lower lines are useful for settlement calculations for rigid foundations.

Fig.8. shows that $\gamma H$ varies more as Poisson's ratio $v$ increases. Non-dimensional characteristic values $\gamma H$ are almost constant in the cases of Poisson's ratio $v \leqq$ 0.3 and $0.2 \leqq R / H \leqq 5$. The maximum error ratios of the PM solutions are shown in Fig.9. so that the errors 
Table 1. Non-dimensional Characteristic Values $\gamma H$

Upper line: by the weighted least-squares sum method Lower line: by fitting the maximum displacement

\begin{tabular}{|c|c|c|c|c|c|c|}
\hline $\boldsymbol{R} / \boldsymbol{H}$ & $v=\mathbf{0}$ & $v=\mathbf{0 . 1}$ & $v=0.2$ & $v=\mathbf{0 . 3}$ & $v=\mathbf{0 . 3 5}$ & $v=\mathbf{0 . 4}$ \\
\hline \multirow{2}{*}{0.02} & 5.15 & 5.25 & 5.56 & 6.35 & 7.24 & 9.02 \\
\hline & 7.14 & 7.37 & 7.80 & 8.86 & 9.70 & 11.52 \\
\hline \multirow{2}{*}{0.05} & 4.73 & 4.84 & 5.15 & 5.89 & 6.72 & 8.43 \\
\hline & 5.74 & 5.90 & 6.27 & 7.08 & 7.93 & 9.65 \\
\hline \multirow{2}{*}{0.1} & 4.38 & 4.49 & 4.79 & 5.54 & 6.39 & 8.17 \\
\hline & 4.92 & 5.13 & 5.43 & 6.24 & 7.11 & 9.03 \\
\hline \multirow{2}{*}{0.2} & 4.10 & 4.23 & 4.55 & 5.38 & 6.32 & 8.41 \\
\hline & 4.29 & 4.54 & 4.90 & 5.78 & 6.84 & 9.48 \\
\hline \multirow{2}{*}{0.5} & 3.92 & 4.09 & 4.51 & 5.62 & 6.98 & 10.20 \\
\hline & 4.08 & 4.27 & 4.73 & 6.05 & 8.04 & 16.82 \\
\hline \multirow{2}{*}{1} & 3.89 & 4.11 & 4.61 & 5.94 & 7.60 & 11.61 \\
\hline & 4.00 & 4.23 & 4.75 & 6.57 & 10.19 & 14.02 \\
\hline \multirow{2}{*}{2} & 3.90 & 4.20 & 4.67 & 6.05 & 7.70 & 11.56 \\
\hline & 4.00 & 4.18 & 4.81 & 6.98 & 12.34 & —1 \\
\hline \multirow{2}{*}{5} & 3.92 & 4.16 & 4.70 & 5.97 & 7.39 & 10.38 \\
\hline & 3.99 & 4.21 & 4.82 & 7.18 & 13.49 & —1 \\
\hline
\end{tabular}

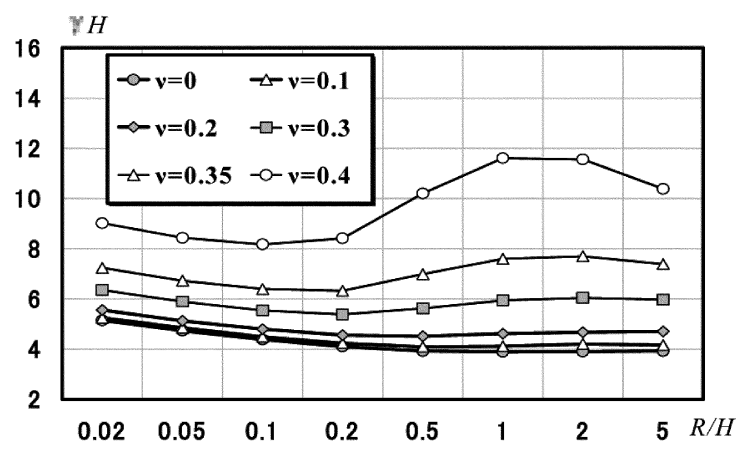

Fig.8. Distribution of $\gamma H$

can be checked against the values of the upper lines in Table 1. The errors are estimated by the ratio of the displacement error to the maximum displacement. According to Fig.9., the maximum error ratios are not more than $9 \%$ when Poisson's ratio $v \leqq 0.3$ and $0.2 \leqq$ $R / H \leqq 5$, and they increase greatly when $R / H<0.2$ or $v>0.3$.

The recommended value of $\gamma H$ for every Poisson's ratio is proposed to be between $0.2 \leqq R / H \leqq 5$ when Poisson's ratio is $v \leqq 0.3$, which is convenient for practical use. Of course, the values in Table 1. will produce better approximations for specific problems. Thus, Table 2. shows the recommended values of $\gamma H$ for an approximation with a $9 \%$ error ratio. Based on these recommended values, the maximum error ratios are shown in Fig.10. For reference, the maximum error ratios in the cases of Poisson's ratios $v=0.35$ and 0.4 are included in Fig. 10. This figure shows that the maximum error ratios are almost the same as those in Fig.9.

Consequently, modified formulas are again presented in Eqs. (26) and (27) for surface displacement calculations. An approximate calculation is realized using Eq. (26) in conjunction with the recommended non-dimensional characteristic value $\gamma H$ in Table 2 . The accuracy is within the $10 \%$ total error ratio even if the FEM solution has a $1 \%$ error ratio.

$$
\begin{array}{ll}
0 \leq r \leq R: & W=S \frac{K_{0}(\gamma R)}{K_{2}(\gamma R)} \\
r>R: & W=S \frac{K_{0}(\gamma r)}{K_{2}(\gamma R)}
\end{array}
$$

where

$S=\frac{q}{k}=\frac{(1+v)(1-2 v) q H}{(1-v) E}$

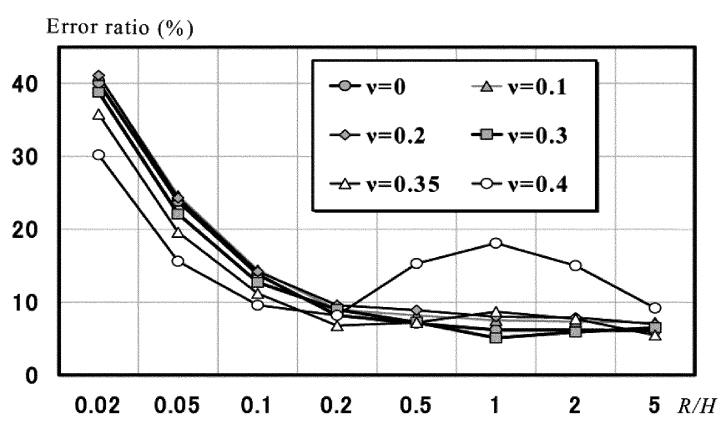

Fig.9. Error ratios of PM

(due to $\gamma H$ by the weighted least-squares sum method)

Table 2. Recommended Non-dimensional Characteristic Values $\gamma H$

\begin{tabular}{ccccc}
\multicolumn{5}{c}{$(v \leqq 0.3$ and $0.2 \leqq R / H \leqq 5)$} \\
\hline Poisson's ratio & $\boldsymbol{v}=0$ & $\boldsymbol{v}=0.1$ & $\boldsymbol{v}=0.2$ & $\boldsymbol{v}=0.3$ \\
$R / H$ & 4.1 & 4.2 & 4.5 & 5.4 \\
\hline
\end{tabular}

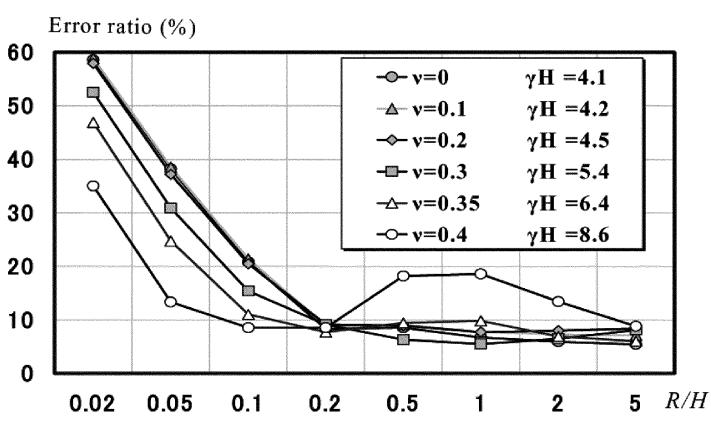

Fig.10. Error ratios of PM

(due to recommended $\gamma H$ except $\boldsymbol{v}>0.3$ )

\section{Limits of PM Applicability}

As for the limits of PM application, soils having Poisson's ratio of $v \geqq 0.35$ are mostly cohesive and mainly consolidated, so an elastic linear analysis is not appropriate. Such soils need further research into their consolidation mechanisms.

There are other reasons for the limits of PM application. One is that, in general, horizontal displacements inside a continuum subjected to vertical loads increase if Poisson's ratio $v$ increases. These 
actual behaviors disprove Vlasov's assumptions that horizontal displacements can be neglected. Another is that Vlasov's assumptions contain only one vertical displacement profile and yet, generally, vertical displacement profiles are not the same in a continuum except when $R \rightarrow \infty$. In particular, when $R / H$ becomes small and the load condition approaches that of a concentrated load, the strain distributions beneath the loading area in the continuum differ greatly from those of Vlasov's assumptions. As a result, the error ratios increase. If $R / H$ becomes large, the strain distributions approach Vlasov's assumptions and the error ratios decrease. Therefore, the applicability limits of $v \leqq$ 0.3 and $0.2 \leqq R / H \leqq 5$ are concluded for a good approximation.

For example, non-dimensional displacements $W / S_{0}$ of the PM are plotted in Fig.11. with those of the FEM in case of the recommended $\gamma H$ value of 5.4 for Poisson's ratio $v=0.3$. Fig. 11 . shows that the solutions obtained from the PM are in good agreement with those of the FEM.

Finally, the closed-form formulation in Eqs. (26) and (27) are useful for wide-ranging mechanical interaction analyses of continua.

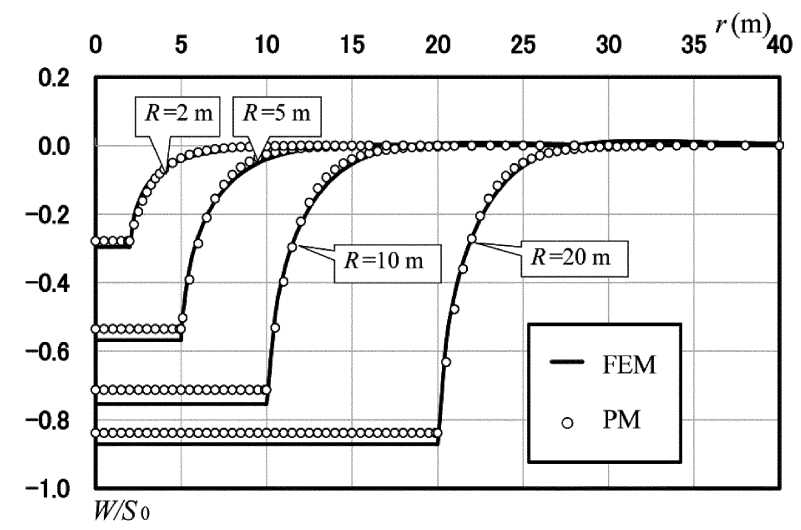

Fig.11. Comparison of PM with FEM $(\gamma H=5.4, v=0.3)$

\section{Conclusions}

The major conclusions are summarized as follows:

1) The reaction of the PM underneath the rigid foundation was shown by the Lagrange multiplier method. The reaction corresponds to the concentrated edge reaction of a rigid plate on an elastic half space.

2) A simple closed-form formulation of surface displacement distributions and the non-dimensional characteristic values of the Pasternak model are proposed for a rigid circular foundation on an elastic layer with a finite depth.

3) To obtain a level of accuracy at which the error ratios are less than $10 \%$, the non-dimensional characteristic values $\gamma H$ necessary for numerical calculation are recommended as constant values between 4.1 and 5.4, depending only on Poisson's ratios. These values are almost the same as those of cases previously studied.

4) The proposed method is convenient for practical analyses of rigid foundations, such as preliminary analyses, order checking, predicting differential settlements, etc., although the applicable conditions are limited to the elastic layers of Poisson's ratio $v \leqq 0.3$ and $0.2 \leqq R / H \leqq 5$. The closed-form formulation of rigid foundations also can be applied to wide-ranging mechanical interaction analyses.

\section{Acknowledgment}

The author would like to express deep appreciation and gratitude to Professor D. Novak of Brno University of Technology, Czech Republic, for providing useful information.

\section{References}

1) Filonenko-Borodich, M.M. (1940) Some approximate theories of elastic foundation, Uchenyie Zapiski Moskovskogo Gosudarstuennogo Universiteta Mekhanika, Moscow, 46, pp.3-18 (in Russian).

2) Hetényi, M. (1946) Beams on elastic foundation, The University of Michigan Press, Ann Arbor.

3) Jones, R., and Xenophontos, J. (1977) The Vlasov foundation model. Int. J. Mech. Sci., 19, pp.317-323.

4) Kerr, A.D. (1964) Elastic and viscoelastic foundation models, J. Appl. Mech., 31, pp.491-498.

5) Loof, H.W. (1965) The theory of the coupled spring foundation as applied to the investigation of structures supported on soil, Heron, Delft, 3, pp.29-49.

6) Pasternak, P.L. (1954) On a new method of analysis of an elastic foundation by means of two foundation constants, Gosudarstvennoe Izdatelstvo Literaturi po Stroitelstuve i Arkhitekture, Moscow (in Russian).

7) Reissner, E. (1958) A note on deflections of plates on a viscoelastic foundation, J. Appl. Mech., ASME, 25, pp.144-145.

8) Tanahashi, H. (2000a) Two-dimensional analysis of ground surface displacements using Pasternak Model, J. Struct. Constr. Engrg., AI J, 530, pp.85-91 (in Japanese).

9) Tanahashi, H. (2000b) Axisymmetric static analysis of surface displacements of a three-dimensional elastic layer on a rigid base using Pasternak Model, J. Struct. Constr. Engrg., AIJ, 531, pp.101-107 (in Japanese).

10) Tanahashi, H. (2001) Characteristics of rigid foundations on Pasternak Model (In case of two-dimensional plane strain condition), Summaries of Technical Papers of Annual Meeting, AIJ, pp.519-520 (in Japanese).

11) Vallabhan, C.V.G., and Das, Y.C. (1988) Parametric study of beams on elastic foundations, J. Engrg. Mech., ASCE, 114(12), pp.2072-2082.

12) Vallabhan, C.V.G., and Das, Y.C. (1991) Analysis of circular tank foundations, J. Engrg. Mech., ASCE, 117(4), pp.789-797.

13) Vlasov, V.Z. and Leont'ev, N.N. (1966) Beams, plates and shells on elastic foundations, Israel Program for Scientific Translations, Jerusalem (Translated from Russian; original Russian version published in 1960).

14) Washizu, K. (1975) Variational methods in elasticity and plasticity, Second edition, Pergamon Press.

15) Winkler, E. (1867) Die Lehre von der Elastizität und Festigkeit, H. Dominicus, Prague (in German) 